\title{
An Examination of the Present State of the Grimsby Trawl Fishery, with especial reference to the Destruction of Immature Fish." Revision of Tables.
}

Aвоuт a year subsequent to the appearance of my paper on the Grimsby Trawl Fishery my attention was directed to certain arithmetical errors in the table on pp. 406 and 407 (pp. 70 and 71 of the Reprint). These errors are in truth, considerable, though fortunately not of a nature to affect the arguments brought forward in the text, as will appear to such of my readers as may be at pains to compare the revised edition with the original.

Although I have no intention of seeking to evade the responsibility for the figures published under my name, I may ask, nevertheless, to be allowed to advert briefly to the circumstances under which they went to press. At the time my paper was in preparation I was suffering from an illness that ultimately compelled me, with much regret, to sever my connection with the Association; and when it seemed advisable to expand my original statistics of "boxes" into columns of other quantities, I found my eyesight unequal to the cyphering thereby entailed. I was therefore obliged to confide the calculations, in great part, to other hands, with results sufficiently disastrous to my own reputation for accuracy.

The revision of the tables has brought to light two errors, which are not those of arithmetic, and for which the responsibility is entirely my own. In the entries for June, July, and August, 1892, it has been explained that 1000 cwt. was subtracted from the Board of Trade returns, as representing approximately the quantity of fish landed from Iceland during the said months. My intention, though not clearly explained in the text, was to subtract the amount from the aggregate; but in the tables it was inadvertently taken from each separate month.

* Journ. Mar. Biol. Assoc., vol. iii. No. 5, Special Number, pp. 339-448. Reprinted under the above title. 1895 . 
In the present edition this item will be found to have been altered in accordance with the actual conditions, the amount subtracted being divided between the three months in proportions which correspond roughly with the relative abundance of Iceland fish during the period concerned. The figures 49,000 in column i were a misprint for 41,000 -the Board of Trade total. A further error appears to have been made in the number of boxes for June, 1893, and this has accordingly been altered to the number originally published in the Journal of the Association, vol, iii. p. 124.

The table on p. 410 (Reprint, p. 74) is dependent on the calculations in the former table, and is therefore vitiated by the errors alluded to. It happens, however, that the revision of this table only brings into greater prominence the destruction of undersized fish.

My attention has recently been drawn to the absence of any definite statement in my text as to the method by which the averages of number of fish in boxes of different qualities were deduced. At this lapse of time I regret that I am unable to lay hands upon the original figures, and can only state that I deduced averages from the contents of a large number of boxes of each quality counted during the earlier period of my work, and checked the results so obtained from time to time during the later years; and in order to run no risk of exaggerating the proportion of undersized fish, I actually made use of averages which somewhat underestimated such proportion.

It remains for me to express my indebtedness to the Association for publishing this corrected version of my tables (which my own circumstances did not allow of my undertaking), and to Professor Weldon and Mr. E. J. Allen for the revision of my figures. 


\section{Table showing the Weight, Bulk, and approximate Number of Plaice fishing power (in voyages of steam-trawlers)}

The terms "large" and "small" in this table refer only to the market designation under

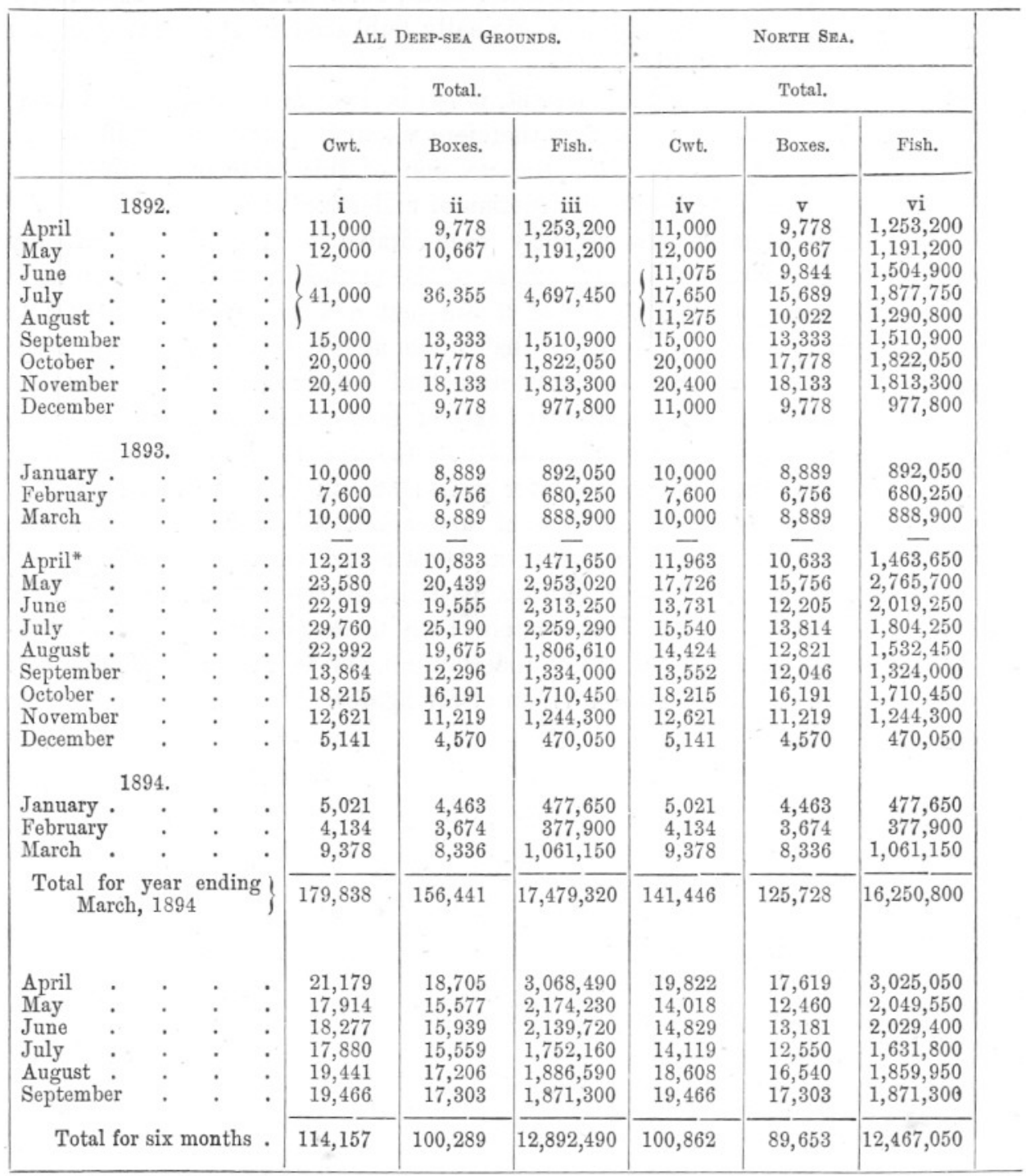

* Totals previous to this date are taken from official returns. 
landed at Grimsby by deep-sea trawlers, and (col. xvi) the diversion of from the North Sea grounds in each month.

which the fish so enumerated are sold. Vide text, pp. 402 and 403 (pp. 66 and 67 of Reprint).

\begin{tabular}{|c|c|c|c|c|c|c|c|c|c|}
\hline \multicolumn{6}{|c|}{ North SEA. } & \multicolumn{4}{|c|}{ ICELAND. } \\
\hline \multicolumn{3}{|c|}{ Large. } & \multicolumn{3}{|c|}{ Small. } & \multirow{2}{*}{ Cwt. } & \multirow{2}{*}{ Boxes. } & \multirow{2}{*}{ Fish. } & \multirow{2}{*}{ Voyages. } \\
\hline Cwt. & Boxes. & Fish. & Cwt. & Boxes. & Fish. & & & & \\
\hline vii & viii & ix & $\mathrm{x}$ & xi & xii & xiii & xiv & $x V$ & xvi \\
\hline 8,935 & 7,942 & 794,200 & 2,066 & 1,836 & 459,000 & .. & $\ldots$ & Av & $\ldots$ \\
\hline 11,067 & 9,837 & 983,700 & 934 & 830 & 207,500 & $\ldots$ & $\cdots$ & $\cdots$ & $\cdots$ \\
\hline 7,171 & 6,374 & 637,400 & 3,904 & 3,470 & 867,500 & $\ldots$ & $\ldots$ & $\ldots$ & $\ldots$ \\
\hline 15,334 & 13,630 & $1,363,000$ & 2,316 & 2,059 & 514,750 & 1,000 & 800 & 24,000 & $?$ \\
\hline 9,110 & 8,098 & 809,800 & 2,165 & 1,924 & 481,000 & ) $\ldots$ & $\ldots$ & $\ldots$ & $\ldots$ \\
\hline 13,668 & 12,149 & $1,214,900$ & 1,332 & 1,184 & 296,000 & $\ldots$ & $\cdots$ & $\cdots$ & $\cdots$ \\
\hline 19,668 & 17,483 & $1,748,300$ & 332 & 295 & 73,750 & $\ldots$ & $\ldots$ & $\cdots$ & $\cdots$ \\
\hline 20,400 & 18,133 & $1,813,300$ & $\ldots$ & $\ldots$ & $\ldots$ & $\ldots$ & $\cdots$ & $\cdots$ & $\cdots$ \\
\hline 11,000 & 9,778 & 977,800 & $\cdots$ & $\cdots$ & $\cdots$ & $\cdots$ & $\cdots$ & $\cdots$ & $\cdots$ \\
\hline 9,977 & 8,868 & 886,800 & 24 & 21 & 5,250 & $\cdots$ & $\cdots$ & $\cdots$ & $\cdots$ \\
\hline 7,566 & 6,725 & 672,500 & 35 & 31 & 7,750 & $\ldots$ & $\ldots$ & $\ldots$ & $\ldots$ \\
\hline 10,000 & 8,889 & 888,900 & $\cdots$ & $\cdots$ & $\ldots$ & $\cdots$ & $\cdots$ & $\cdots$ & $\cdots$ \\
\hline$\overline{80}$ & - & $70 \overline{1}$ & $\overline{3} 0$ & $\overline{2}_{669}$ & $6 \overline{7} 250$ & $\overline{7}_{250}$ & $\overline{2}_{200}$ & $\overline{8} 000$ & $\overline{2}$ \\
\hline 8,960 & 7,964 & 796,400 & 3,003 & 2,669 & 667,250 & 250 & 200 & 8,000 & 2 \\
\hline 8,800 & 7,822 & 782,200 & 8,926 & 7,934 & $1,983,500$ & 5,854 & 4,683 & 187,320 & 20 \\
\hline 7,740 & 6,880 & 688,000 & 5,991 & 5,325 & $1,331,250$ & 9,188 & 7,350 & 294,000 & 30 \\
\hline 12,369 & 10,995 & $1,099,500$ & 3,171 & 2,819 & 704,750 & 14,220 & 11,376 & 455,040 & 36 \\
\hline 12,546 & 11,152 & $1,115,200$ & 1,878 & 1,669 & 417,250 & 8,568 & 6,854 & 274,160 & 21 \\
\hline 12,656 & 11,250 & $1,125,000$ & 896 & 796 & 199,000 & 312 & 250 & 10,000 & 1 \\
\hline 17,530 & 15,582 & $1,558,200$ & 685 & 609 & 152,250 & $\ldots$ & $\ldots$ & $\ldots$ & $\ldots$ \\
\hline $\mathrm{b1}, 703$ & 10,403 & $1,040,300$ & 918 & 816 & 204,000 & $\ldots$ & $\cdots$ & $\cdots$ & $\cdots$ \\
\hline 5,043 & 4,483 & 448,300 & 98 & 87 & 21,750 & $\cdots$ & $\cdots$ & $\cdots$ & $\cdots$ \\
\hline 4,786 & 4,254 & 425,400 & 235 & 209 & 52,250 & $\cdots$ & $\cdots$ & $\cdots$ & $\cdots$ \\
\hline 4,055 & 3,604 & 360,400 & 79 & 70 & 17,500 & $\ldots$ & $\cdots$ & $\cdots$ & $\cdots$ \\
\hline 7,671 & 6,819 & 681,900 & 1,707 & 1,517 & 379,250 & $\cdots$ & $\cdots$ & $\cdots$ & $\cdots$ \\
\hline 113,859 & 101,208 & $10,120,800$ & 27,587 & 24,520 & $6,130,000$ & 38,392 & 30,713 & $1,228,520$ & 110 \\
\hline 10,348 & 9,198 & 919,800 & 9,474 & 8,421 & $2,105,250$ & 1,357 & 1,086 & 43,440 & 7 \\
\hline 7,991 & 7,103 & 710,300 & 6,027 & 5,357 & $1,339,250$ & 3,896 & 3,117 & 124,680 & 25 \\
\hline 9,494 & 8,439 & 843,900 & 5,335 & 4,742 & $1,185,500$ & 3,448 & 2,758 & 110,320 & 21 \\
\hline 11,293 & 10,038 & $1,003,800$ & 2,826 & 2,512 & 628,000 & 3,761 & 3,009 & 120,360 & 19 \\
\hline 17,063 & 15,167 & $1,516,700$ & 1,545 & 1,373 & 343,250 & 833 & 666 & 26,640 & 5 \\
\hline 18,408 & 16,363 & $1,636,300$ & 1,058 & 940 & 235,000 & $\cdots$ & $\cdots$ & $\cdots$ & $\cdots$ \\
\hline 74,597 & 66,308 & $6,630,800$ & 26,265 & 23,345 & $5,836,250$ & 13,295 & 10,636 & 425,440 & 77 \\
\hline
\end{tabular}


Table showing the Numbers and Proportion of Plaice of different sizes landed at Grimsby by deep-sea trawlers in one year.

\begin{tabular}{|c|c|c|c|c|c|c|c|c|c|c|}
\hline & \multirow{3}{*}{. } & & \multicolumn{4}{|c|}{ Sexually } & \multirow{2}{*}{\multicolumn{2}{|c|}{$\begin{array}{l}13 \text { inches and } \\
\text { above. }\end{array}$}} & \multirow{2}{*}{\multicolumn{2}{|c|}{$\begin{array}{c}\text { Below } \\
13 \text { inches. }\end{array}$}} \\
\hline & & & \multicolumn{2}{|c|}{$\begin{array}{c}\text { Mature. } \\
17 \text { inches and a bove. }\end{array}$} & \multicolumn{2}{|c|}{$\begin{array}{l}\text { Immature. } \\
\text { Below } 17 \text { inches. }\end{array}$} & & & & \\
\hline & & & No. & $\%$ & No. & $\%$ & No. & $\%$ & No. & $\%$ \\
\hline 1894. & $\begin{array}{l}\text { April : } \\
\text { May }: \\
\text { June }: \\
\text { July }: \\
\text { August } \\
\text { September } \\
\text { October } \\
\text { November } \\
\text { December } \\
\text { January } \\
\text { February } \\
\text { March. }\end{array}$ & $\dot{\dot{i}} \dot{.}$. & $\begin{array}{c}\mathrm{i} \\
557,480 \\
547,540 \\
481,600 \\
769,650 \\
780,640 \\
787,500 \\
1,090,740 \\
728,210 \\
313,810 \\
297,780 \\
252,280 \\
477,330\end{array}$ & $\begin{array}{l}\mathrm{ii} \\
38 \\
20 \\
24 \\
43 \\
51 \\
59 \\
64 \\
59 \\
67 \\
62 \\
67 \\
45\end{array}$ & $\begin{array}{r}\text { iii } \\
906,170 \\
2,218,160 \\
1,537,650 \\
1,034,600 \\
751,810 \\
536,500 \\
619,710 \\
516,090 \\
156,240 \\
179,870 \\
125,620 \\
583,820\end{array}$ & $\begin{array}{l}\text { iv } \\
62 \\
80 \\
76 \\
57 \\
49 \\
41 \\
36 \\
41 \\
33 \\
38 \\
33 \\
55\end{array}$ & $\begin{array}{c}\mathbf{v} \\
783,485 \\
902,330 \\
752,325 \\
1,060,025 \\
1,045,405 \\
1,032,400 \\
1,417,605 \\
956,670 \\
405,645 \\
388,085 \\
326,110 \\
651,635\end{array}$ & $\begin{array}{l}\text { vi } \\
53 \\
33 \\
37 \\
59 \\
68 \\
78 \\
83 \\
77 \\
86 \\
81 \\
86 \\
61\end{array}$ & $\begin{array}{r}\text { vii } \\
680,165 \\
1,863,370 \\
1,266,925 \\
744,225 \\
487,045 \\
291,600 \\
292,845 \\
287,630 \\
64,405 \\
89,565 \\
51,790 \\
409,515\end{array}$ & $\begin{array}{l}\text { viii } \\
47 \\
67 \\
63 \\
41 \\
32 \\
22 \\
17 \\
23 \\
14 \\
19 \\
14 \\
39\end{array}$ \\
\hline Tot & 1 for year & . & $7,084,560$ & 44 & $9,166,240$ & 56 & $9,721,720$ & 60 & $6,529,080$ & 40 \\
\hline
\end{tabular}

